Fanum

Sociológico

\section{Forum Sociológico}

Série II

23 | 2013

Número 23

\title{
De lo sórdido a lo vintage, de la marginalización a la distinción. Gentrificación y ocio nocturno en Cais do Sodré, Lisboa
}

Jordi Nofre

\section{(2) OpenEdition \\ Journals}

Edição electrónica

URL: https://journals.openedition.org/sociologico/827

DOI: $10.4000 /$ sociologico. 827

ISSN: 2182-7427

Editora

CICS.NOVA - Centro Interdisciplinar de Ciências Sociais da Universidade Nova de Lisboa

Edição impressa

Data de publição: 1 novembro 2013

Paginação: 59-67

ISSN: 0872-8380

Refêrencia eletrónica

Jordi Nofre, «De lo sórdido a lo vintage, de la marginalización a la distinción. Gentrificación y ocio nocturno en Cais do Sodré, Lisboa», Forum Sociológico [Online], 23 | 2013, posto online no dia 01 janeiro 2014, consultado o 30 março 2022. URL: http://journals.openedition.org/sociologico/827 ; DOI: https://doi.org/10.4000/sociologico.827

Este documento foi criado de forma automática no dia 30 março 2022.

(c) CICS.NOVA 


\section{De lo sórdido a lo vintage, de la marginalización a la distinción. Gentrificación y ocio nocturno en Cais do Sodré, Lisboa}

Jordi Nofre

\section{Introducción}

1 A lo largo de las últimas décadas, las ciudades postfordistas han sido objeto de numerosas estrategias culturales para la renovación urbano no tan sólo de la ciudad central sino también de sus suburbios. Las nuevas formas de organización social, ocio y consumo cultural han conducido recientemente a la conversión tanto de los centros históricos como de las áreas de nueva centralidad urbana en "parques temáticos" urbanos (Lefebvre, 1968; Edensor, 1998; Hannigan, 1998; Gottdiener, 2001, Judd, 2003; Baptista, 2005). En este proceso, el ocio nocturno juega un papel fundamental, contribuyendo a su vez en el proceso de internacionalización de la ciudad y de construcción de su marca urbana (Gottdiener, 2001; Chatterton y Hollands, 2003; Nofre, 2009a). En este artículo se presentan los primeros resultados derivados de un trabajo de campo cualitativo aún en marcha acerca de la relación entre gentrificación y ocio nocturno en Lisboa (Portugal). Concretamente, este texto se centra en el estudio de Cais do Sodré (una de las partes del centro histórico postpombalino de Lisboa) en donde la conversión de la Pensão Amor (hostal abandonado y anteriormente frecuentado por los prostitutas "marginales" y marineros desde inicios del siglo $\mathrm{XX}$ ) está actualmente jugando un papel clave en la gentrificación del antiguo barrio marinero de Cais do Sodré a partir de la promoción de una "noche" inundada de motivos retro y vintage como elementos de distinción social para ser consumidos por las clases medias altas tanto locales como globales. En este sentido, y como se verá a lo largo del artículo, las tradicionales teorías sobre la renda y el valor del suelo en la explicación de los procesos de gentrificación si bien pueden ser útiles en el análisis geográfico de la gentrificación 
en un período largo, parecen ser insuficientes en el análisis etnográfico del día a día del proceso gentrificador.

El trabajo de campo llevado a cabo concretamente en el área de Cais do Sodré se inició en Octubre de 2011 - si bien en Enero de 2010 ya se iniciaron observaciones no sistematizadas con relativa frecuencia. Esta primera fase de trabajo observacional finalizó a finales de Diciembre de 2012. La segunda fase, de carácter comparativo con otras áreas de la capital portuguesa, sigue su curso en la actualidad. De hecho, el trabajo de campo ha tenido dos fases en lo que se refiere a su continuidad: 1) Del 5/10/2011 al 10/01/2012 y el 10/01/2012, y del 3/10/2011 al 9/01/2012, el trabajo de campo se realizó de manera discontínua por la imposibilidad de estar en la ciudad varias semanas por motivos profesionales; 2) durante el período de siete meses comprendidos entre el 10/01/2012 y el 29/09/2012, el trabajo de campo se llevó a cabo ininterrumpidamente todos los míercoles, jueves, viernes y sábado de cada semana. El día 23 de Diciembre de 2012 se inició la redacción final de este artículo.

Para este estudio eminentemente cualitativo sobre la noche de Cais do Sodré han sido tomados en consideración el conjunto de locales situados en la calle Rua Nova do Carvalho entre Travessa dos Remolares y Rua de São Paulo. Los locales que han sido "monitorizados" para este estudio han sido Pensão Amor (local principal de análisis), Bar da Velha Senhora, Sol e Pesca, Bar do Cais, Roterdão, Musicbox, Liverpool, Oslo Bar, Europa, Tokyo, Jamaica y Viking. La monitorización de los locales incluyó también el "área de influencia" de cada local en la calle - pues se permite beber (noble acto de sociabilidad) en el espacio público. Como resultado de ello, tanto los procesos de observación flotante como algunas de las entrevistas tuvieron lugar en la misma calle Rua Nova do Carvalho, en su tramo correspondiente entre Rua de São Paulo y el puente de Rua do Alecrim.

La complejidad del fenómeno estudiado así como las prácticamente nulas referencias teórico-metodológicas anteriores a este estudio que se presenta ${ }^{1}$ - las cuales podrían haber servido de punto de partida para este trabajo - sugirieron al autor de este artículo la adopción del denominado "eclecticismo metodológico" (Hannerz, 1980; Wynne, 1998) como marco metodológico más apropiado. De ahí que, a partir de la aplicación de una metodología eminentemente transductiva - mediante la cual se elabora y se construye un objeto teórico posible a partir de informaciones relativas a la realidad y de una problemática planteada por esta realidad (Lefebvre, 1968) -, se hayan utilizado un elenco de técnicas cualitativas de estudio, como la observación flotante (Petónnet, 1982); la observación participante y la observación "intrusiva" (Russell, 2008); las anotaciones en primera persona acerca de lo observado; y, por último, las entrevistas informales. Los 32 individuos entrevistados fueron 4 miembros de seguridad de los locales (todos ellos hombres, de entre 35 y 45 años, de nacionalidad portuguesa); 3 policías, hombres, dos de ellos entre 35 y 45 años y el tercero entre 50 y 60 años (uno de ellos jefe de escuadrón en Lisboa), y 25 clientes (14 chicas de entre 22 y 38 años, de las cuales 6 eran estudiantes Erasmus; y 11 chicos de entre 25 y 38 años; de los cuales solamente ${ }^{2}$ eran Erasmus). A todo ello cabe sumarle el hecho que la totalidad de los propietarios de los locales de Cais do Sodré no han querido participar en esta investigación, al menos hasta la fecha. Por otra parte, cabe mencionar que los 4 miembros de seguridad fueron contactados a partir de uno de ellos con el que establecí una cierta relación de confianza: 2 de ellos pertenecían al mismo local (evidentemente no daré nombres concretos de locales para preservar su anonimato tal y como me lo 
solicitaron explícitamente). Las entrevistas con ellos tuvo lugar entre Mayo y Junio de 2012. Los tres policías (uno de ellos es un habitual "secreta" de la zona de ocio nocturno de Cais do Sodré) fueron entrevistados días después de sufrir una agresión en pleno trabajo de campo: cosas del oficio... Muy amablemente accedieron a ser entrevistados informalmente pocos días después de tal desgraciado episodio, a inicios de Junio de 2012, en las inmediaciones del área de fiesta de Cais do Sodré, pocos minutos antes del inicio de su ronda de vigilancia. Por otra parte, los clientes fueron abordados y entrevistados in situ, es decir, dentro de los locales o bien en las inmediaciones de la puerta de entrada, en la misma Rua Nova do Carvalho. El registro de la conversa fue realizado memorísticamente para posteriormente, una vez en la barra, en el lavabo o de camino de vuelta a casa, ser volcado en la libreta de campo. Todas las entrevistas informales con los clientes tuvieron lugar entre Marzo de 2012 e inicios de Diciembre de 2012, pocas semanas antes del inicio de la redacción de este artículo. Como última nota metodológica, cabe destacar que la verificación de las diferentes informaciones obtenidas a partir tanto de las entrevistas informales como del trabajo de campo observacional fueron verificadas a partir de la triangulación establecida entre agentes de seguridad, clientes y anotaciones derivadas del trabajo de campo observacional. Cada información surgida en cada uno de estos ámbitos fue introducida en las diferentes entrevistas informales llevadas a cabo con individuos de los tres grupos de informantes citados (agentes de seguridad, policía y clientes) para proceder a su verificación o bien a su contraposición, emergiendo de ahí visiones diferentes acerca de los temas "críticos" tratados como la segregación socioespacial (y racial) de la noche en Cais do Sodré y sus mecanismos de ejecución.

Figura 1 粮 Localización del área de estudio

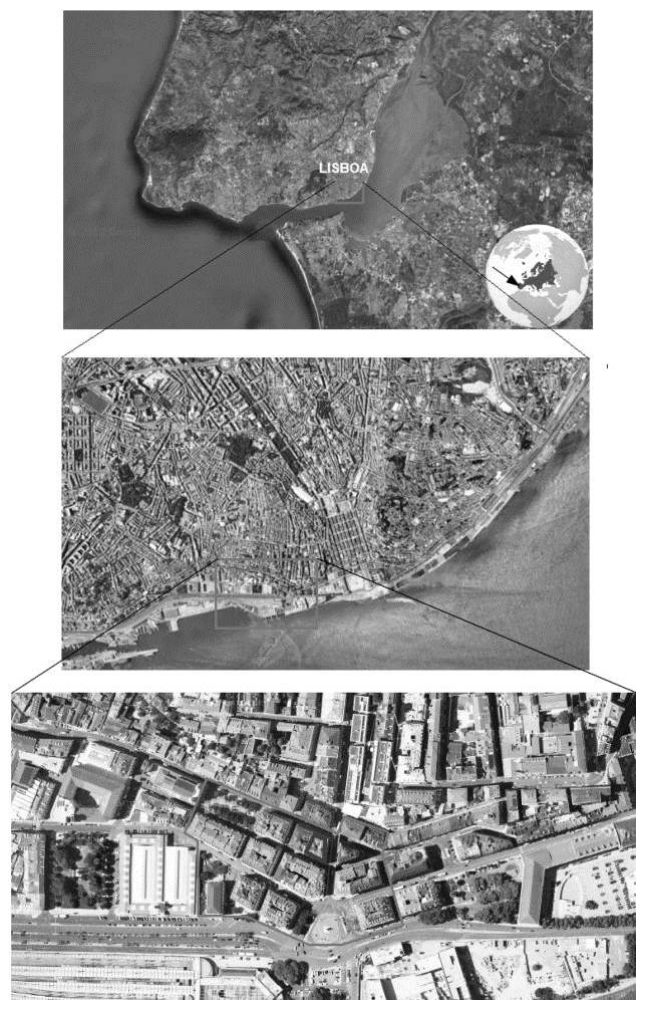

Fuente: Nofre, J. (2013). Base ortofotográfica cortesía de Google Maps @ 2013 
5 Antes de proceder a presentar cómo el consumo de "lo vintage" en el ámbito de un ocio nocturno elitista forma parte de las estrategias de higienización social de barrios de clase trabajadora del centro de Lisboa, se antoja como necesario un breve encuadramiento teórico acerca de la relación entre ocio nocturno y gentrificación que, ciertamente, puede resultar muy útil en la posterior lectura geográfica de este proceso de transformación urbana de un sector del centro histórico de la capital portuguesa.

\section{El ocio nocturno como estratégia gentrificadora: breves notas teóricas}

6 No cabe duda que, junto con las teorías diferenciales de la renda (Neil Smith, 1979) y del valor del suelo (Hamnett y Randolph, 1984, 1986), la cultura y su consumo se han convertido en aspectos fundamentales en la explicación de cómo ha evolucionado la gentrificación en las ciudades de todo el mundo a lo largo del último cuarto de siglo. ${ }^{2} \mathrm{~A}$ pesar de ello, el estudio del ocio nocturno como "estrategia gentrificadora" ha recibido poca atención hasta la fecha a pesar de la existencia, veinte años atrás, de algunos estudios que ya sugerían explorar con más detalle tal relación ante la emergencia de un nuevo estilo de vida basado en la distinción social propia de las nuevas clases medias urbanas de las ciudades postindustriales (Thornton, 1995; Savage y Butler, 1995; Butler, 1997; Wynne y O'Connor, 1998; Ley, 2003; Chatterton y Hollands, 2003). De hecho, la estetización de la vida cotidiana en las sociedades postindustriales debe ser considerada como un proceso clave en la (re)producción de un ocio nocturno "distinguido" (Nofre y Martin, 2009; Nofre, 2009a; 2011), socialmente higienizado y partícipe de una gentrificación que sin duda alguna condiciona el espacio físico urbano a la reproducción de la distinción social y la acumulación de capital cultural y social - en términos de Pierre Bourdieu (1979).

7 Por otra parte, el enfoque espacial para el estudio del ocio nocturno ha venido ganando importancia, haciendo hincapié en la regulación y normativización del consumo de ocio nocturno y de sus espacios (Chatterton y Hollands, 2003; Talbot, 2004, 2006, 2007; Hobbs et al., 2005; entre otros), en la gentrificación como estrategia de "securitización" de los espacios públicos y privados del sistema de ocio nocturno de las ciudades postindustriales (Thomas y Bromley, 2000; Chatterton et al., 2002; Hael, 2011), o entre la relación entre gentrificación y "securitización" del ocio nocturno como estrategia de distinción social, preservación de la pureza de la comunidad - en términos de Richard Sennett (1970) - y remoralización de la juventud (Nofre, 2009b, 2011). Entre este grupo de trabajos cabe destacar los de Paul Chatterton y Robert Hollands $(2002,2003)$ los cuales, entre otros aspectos, ofrecen un análisis detallado de la creciente segregación socioespacial del ocio nocturno en las ciudades de la Europa occidental. Chatterton y Hollands definen lo que denominan como noche "central" y noche "marginal", las cuales presentan, entre ellas, acusadas diferencias en cuanto al consumo de música y tipo de alcohol, estilos de vestir, códigos de comportamiento, etc. Ciertamente, y como afirman los mismos autores, la elitización de la noche comporta una progresiva marginalización espacial (y social) del ocio nocturno de las clases trabajadoras. Sin embargo, ¿es todo lo "antiguo" marginalizado de la "noche" de la ciudad central? 


\section{El consumo de lo vintage como distinción social en la noche global}

8 Uno de los elementos que recientemente ha contribuido más a la acumulación de capital cultural y distinción social en la noche más exclusiva de la ciudad central es el consumo de espacios de ocio nocturno decorados con motivos "vintage", tal y como ha ocurrido en Cais do Sodré (Lisboa). En el caso de la capital portuguesa, este proceso está estrechamente relacionado con la eclosión del movimiento Pin-Up como una nueva forma de consumo hedonista juvenil. En este sentido cabe destacar la abertura en los últimos años de dos tiendas de muebles, elementos de decoración para el hogar, ropa y complementos de vestir de estilo retro y vintage ubicado en el gentrificado Bairro Alto (Mendes 2006): Bad Luck ${ }^{3}$ y Ás de Espadas. La revista Le Cool Lisboa cita esta última tienda como una de las mejores boutiques de moda en Lisboa. ${ }^{4} \mathrm{El}$ estilo vintage y su vuelta a la escena de la moda de gama media-alta está relacionada con ese nuevo consumo hedonista juvenil citado en el párrafo anterior el cual busca, ansiosamente, aquella "autenticidad" que le permita "distinguirse" de la masa de los comunes - tomando prestado el concepto de distinción social acuñado por Pierre Bourdieu (1979).

9 En Lisboa, la oferta de "autenticidad" (en su concepción más genérica) tiene múltiples espacios: desde sesiones de Fado "auténtico" en la Rua dos Remédios, hasta las sardinas a la brasa del arraial de Santo Estêvão. Sin embargo, "lo auténticamente vintage" sólo cuenta con unos pocos espacios de diferente índole dónde puede ser consumido. Tomando la moda de vestir como ejemplo, cabría citar la tienda número 281 que Prada abrió en Junio de 2010 en la glamurosa Avenida da Liberdade. Después de inaugurarse en plena temporada primavera-verano 2010 ofreciendo una línea de productos basados en las playas de todo el mundo, la línea de esta temporada primavera-verano de 2012 está basada en motivos vintage para los complementos de vestir ; en lo que se refiere a las piezas de ropa, para ellos, motivos rockabilly; para ellas, piezas con inspiración retro y pin-up.

10 A la par, el Hotel Flórida 1940 fue re-inaugurado con una fiesta en la que la asociación de animación sociocultural Cais Sodré Cabaret participó invitando, a su vez, a todas aquellas personas que se sintiesen identificadas o les gustase, simplemente, todo aquello relacionado con lo "vintage". A todo ello le ha seguido la recuperación de una escena rockabilly en lo que se refiere a lo musical protagonizada principalmente por los portugueses Texabilly Rockets. Pero sobre todo, y como se ha indicado, Cais Sodré Cabaret juega un papel fundamental en la promoción de lo "vintage" como elemento de acumulación cultural y distinción social en los espacios de ocio nocturno más chics de Lisboa:

“CAIS SODRÉ CABARET! aims to celebrate the times when gentlemen wore hats and ladies wore gloves... a celebration of style and glamour of another epoch. In Portugal, during the 20's and 30's, there were many clubs and cafes that held writers, artists and intellectuals meetings (...). We aim to recreate a retroatmosphere associated to bohemian night life celebration where party and pleasure are priority: music, dancing, drinking and smoking, but also sordid and decadent atmosphere of Cais do Sodré. It was on its dark and dirty alleys, street corners and sidewalks, illuminated by neon light announcing bars with revered city names, where hooker ladies and their pimps, policemen, dockers and all kinds of clients hungry for emotions walked on by, where sailors brought in the first American Rock'n'Roll records. All these spirits are invoked in the party!" ${ }^{5}$ 
11 A comienzo del siglo XX, Cais do Sodré era un barrio de marineros, prostitutas, vino y fado (Machado, 2008) marcado por un ambiente sórdido. Actualmente, esta sordidez ha dado paso a su propia re-significación y conversión en un paisaje de ocio nocturno bohemio-burgués (bourgeoisboho, en su denominación inglesa original); en otras palabras, su conversión en un escenario caracterizado por la presentación de lo "vintage" como símbolo de distinción social. Tal conversión, como se verá en el texto que sigue a continuación, tiene como objetivo higienizar socialmente el barrio de Cais do Sodré, aspecto que forma parte de la reciente estrategia de elitización del centro histórico de Lisboa llevado a cabo por la Câmara Municipal de Lisboa. ${ }^{6}$ El texto que sigue a continuación tiene como objetivo mostrar como la re-significación de la sordidez urbana de Cais do Sodré ha dado paso recientemente a un proceso de elitización de su espacio urbano a través de la promoción de un ocio nocturno global el cual toma elementos decorativos y estéticos "vintage" de aquel ambiente marinero y de prostitución marginal, de tabaco, vino y sexo barato, para construir una nueva escena nocturna orientada a las clases medias-altas de la capital Portuguesa así como a las nuevas clases medias cosmopolitas que están de paso en la ciudad.

\section{Cais do Sodré: de la decadência a una noche gentrificada}

12 La historia urbana de Cais do Sodré está estrechamente relacionada con el crecimiento urbano del frente marítimo de la capital portuguesa de inicios del siglo XVI. Durante el reinado de Manuel I (1495-1521) fueron planificados y desarrollados nuevos espacios riberos que tuvieron como objetivo ampliar las instalaciones portuarias de la ciudad. Víctor Durão (2012) señala que las áreas escogidas para tal desarrollo urbano fueron Santos y Cata-que-Faras (hoy conocida como Cais do Sodré). Una vez que estos terrenos limosos fueron urbanizados, rápidamente acogieron nuevas actividades económicas relacionadas con el tráfico de mercancías portuarias (Durão, 2012). Es por este motivo que la morfología urbana de Cais do Sodré, sus actividades cotidianas y su apropiación por parte de tenderos y comerciantes locales y extranjeros, marineros y prostitutas, tiene sus orígenes en este ajetreado ambiente portuario. Sin embargo, nada de ese pasado sigue existiendo hoy en día. Como la periodista de la BBC Kerry Christiani señala: "Cais do Sodré had upstaged Bairro Alto as Lisbon's most happening nightlife district" (Christiani, 2012). ${ }^{7}$

El "viejo" Cais do Sodré ha dado paso al "nuevo" Cais. Lo que durante muchas décadas atrás era un barrio inundado de alcohol, comida barata, peep shows, música fado, marineros borrachos, episodios de violencia callejera, drogas y prostitución, comenzó a cambiar a mediados de los años 1970, cuando algunas de las tiendas y negocios diversos de Cais do Sodré se reconviertieron en pequeños locales de ocio nocturno, naciendo así locales que aún hoy día siguen abiertos al público, como la Discoteca Jamaica, Bar Europa, Bar Tokyo y Viking. Además otros muchos bares abrieron o bien pasaron a llamarse como los puertos comerciales más famosos del mundo (puerto de Filadelfia, Shangri-La, Tokio, Rotterdam, Copenhague, Liverpool, Oslo, etc). A todo ello cabe sumarle la Revolución de los Claveles de 25 de Abril de 1974, que condujo a la caída del régimen fascista de Salazar y que, en el campo del ocio nocturno, supuso el inicio del proceso de "democratización" de la noche lisboeta. De ahí que Cais do Sodré fuera entonces reapropiado por jóvenes de las clases medias de la ciudad, estudiantes 
universitarios, bohemios, intelectuales y periodistas locales. Sin embargo, tal "democratización", y ya con una perspectiva temporal más amplia, ha comportado un "desplazamiento espacial" de la prostitución marginal. Este proceso presenta hoy día una refuerza vigencia tal y como la periodista Clara Silva (2011) informa:

“Na Pensão Amor ainda há camas, armários, cadeiras e espelhos dos tempos em que o prédio era casa de prostituição. Na verdade, no número 38 da Rua Nova do Carvalho, em Lisboa, funcionavam quatro pensões que alugavam quartos à hora a prostitutas e a marinheiros que atracavam no Cais do Sodré, vindos de várias partes do mundo. Agora, os quartos também podem ser alugados ao dia, à semana ou ao mês, mas a empresas - que nada têm a ver com prostituição."

La Rua do Alecrim desciende desde Largo de Camões hasta Cais do Sodré. Justo antes de llegar, Alecrim cruza a un nivel superior la Rua Nova do Carvalho, la cual constituye el eje vertebrador del clúster de ocio nocturno de Cais do Sodré. Aparte que bajo el puente se encuentre uno de los locales más dinámicos de Lisboa en lo que a programación de eventos musicales se refiere, Musicbox (antiguo Bar Texas) ${ }^{9}$, tal puente divide la noche de Cais do Sodré en dos. En la parte oriental del clúster de Cais do Sodré aún siguen abiertos los pequeños locales de ocio que abrieron a partir de inicios de la década de los setenta y otros que abrieron posteriormente: Jamaica, Viking, Bar Europa, Bar Oslo, Bar Liverpool, Copenhaga, Tokyo. La clientela más o menos fiel - mayoritariamente lisboeta aunque en 2010 los estudiantes Erasmus eran claramente mayoría de Martes a Viernes - responde a su vez a la fidelidad de este tipo de locales mantienen en el cuidado de una noche "auténtica", con una cierta sordidez aunque ni excesiva ni "peligrosa"10 para el cliente: cerveza barata, alcohol blanco de mala calidad, prostitutas baratas, drogas (hachís y cocaína), los mismos temas musicales de siempre (generalmente de género rock y pop-rock de los años 70s, 80s y 90s); el diseño interior de los locales continua siendo el original.

Sin embargo, esta atmósfera algo decadente es re-significada por parte de algunos consumidores (blancos) de clase media-alta los cuales, muchos de ellos, pertenecen al campo profesional de la comunicación y la cultura. ${ }^{11}$ Para algunos de ellos, consumir este tipo de noche "decadente", "cutre" (en términos populares) significa recordar aquellas noches cuando eran más jóvenes. Para otros, significa consumir una "decadencia elitizada", "higienizada", nada "peligrosa", totalmente "controlada": se trata de consumir un ocio nocturno inundado de emociones controladas en un ambiente hiper-securitizado por parte del staff del mismo local así como por un número significativo de policía secreta que opera en Cais do Sodré cada noche. ${ }^{12}$ De hecho, el hecho que la Rua Nova do Carvalho haya sido cerrada al tráfico rodado desde Septiembre de 2011 ha contribuido al reforzamiento de la acción de la seguridad privada de los locales sobre el control del orden público en la calle. Ello supone, sin lugar a dudas, un incremento de la securitización del espacio público, pero también una semiprivatización "encubierta" del ejercicio de la seguridad pública la cual pretende reforzar la ecología del miedo (Davis, 1990) como garante de un orden microsocial establecido a lo largo de los años y que está basado en relaciones de interés entre chulos, pequeños traficantes, carteristas, personal de seguridad de los locales y miembros de la policía. ${ }^{13}$

16 En la parte occidental, la noche es totalmente diferente. En primer lugar porque la presencia de dos agentes de la Polícia de Segurança Pública comporta diferencias radicales sobre quién y cómo se ejerce el control del orden público en la calle. Aquí, los gorilas no extienden sus funciones de vigilancia y control más allá de los límites de su 
local. En segundo lugar, la tipología de oferta de ocio nocturno y de consumidor presenta significativas diferencias gracias a la reciente abertura de tres nuevos locales de ocio nocturno los cuales presentan un diseño interior de marcada tendencia vintage. Son locales que forman parte, como ya ha sido sugerido anteriormente, de una estrategia llevada a cabo por el sector privado en connivencia con la administración pública local para la renovación urbana y social de Cais do Sodré. En este contexto, los locales Pensão Amor, Bar da Velha Senhora, y Sol e Pesca ofrecen un novedoso ocio nocturno basada en el consumo de "lo vintage" y "lo bohemio" como distinción social (Figura 2).

Figura 2 潾 Pensão Amor, fachada desde la calle Rua do Alecrim (arriba a la izquierda), escaleras de acceso a la primera planta (arriba la derecha), performance pocos días después de la inauguración (abajo a la izquierda), y sala principal (abajo a la derecha).
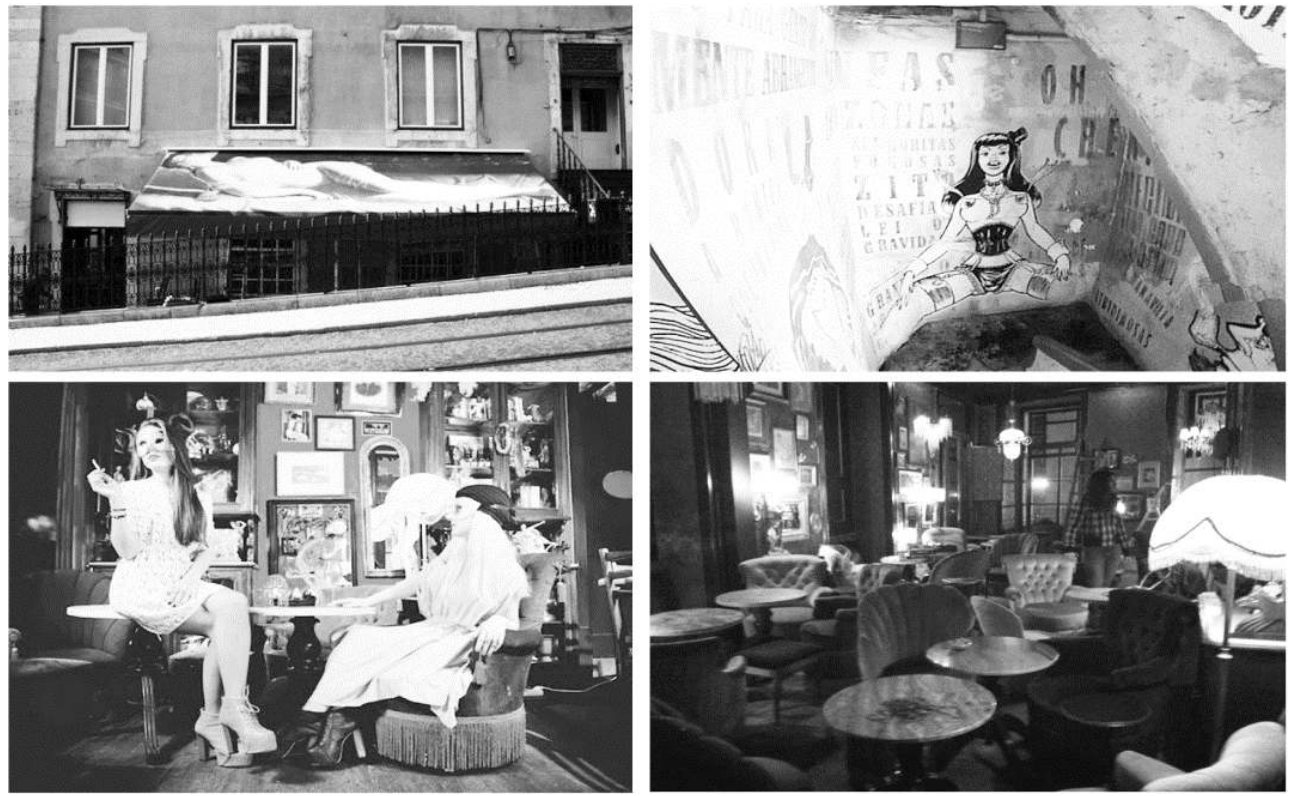

Fuente: Pensão Amor (c) 2012

A pesar de tratar-se claramente de locales destinados a las nuevas clases medias cosmopolitas y a las jóvenes y adultos-jóvenes pertenecientes a las clases medias-altas lisboetas (tal y como deja entrever la petición de vestir bien y los precios del menú de restauración de la Pensão Amor, o los precios de las tapas de Sol e Pesca, por ejemplo), tal "exclusividad" no impide que algunos estudiantes universitarios portugueses y Erasmus (estos últimos, una minoría muy poco significativa) prefieran acudir a estos nuevos locales "gentrificadores" para evitar la sordidez que rodea la "periferia" de este subsector recientemente "higienizado" en términos sociales. ${ }^{14}$

Sin embargo, la reconversión de Cais do Sodré a través de la gentrificación de su ocio nocturno no podría ser completamente entendida sin tener en cuenta el papel fundamental que en este proceso presenta la recientemente inaugurada Pensão Amor. Este nuevo local de ocio nocturno ocupa un viejo edificio de cinco plantas de paredes pintadas de rojo, con lámparas de araña, fotos y dibujos en la pared de chicas salidas de cualquier cabaret o burdel de los años veinte, casi desnudas y en pose erótica, mobiliario vintage, etc. El primer piso de la Pensão Amor presenta un espacio multifuncional en donde se celebran a menudo charlas, presentaciones de libros, conferencias, etc. También hay un espacio donde tomar una copa de pie mientras, con 
un volumen musical muy discreto, el DJ de turno desarrolla su sesión. En el resto de los pisos las antiguas habitaciones de las prostitutas se han transformado en espacios de acogida de iniciativas artísticas y creativas, así como en oficinas de gestión cultural (como la oficina de gestión del Queer Lisboa Festival). Cabe destacar la sala de cabaret en donde la clientela puede leer, charlar y/o beber de manera "distinguida" en un espacio totalmente "higienizado" de cualquier intrusión de clase inferior. Precisamente son los gorilas del local quienes ejecutan tal "higienización social" del local aplicando un criterio de selección de la clientela basado en la catalogación absolutamente arbitraria de etnia "peligrosa" o "no peligrosa" y la apariencia de status quo. En otras palabras, los inmigrantes africanos de las ex colonias portuguesas, los gitanos y todos los que no parecen pertenecer a las clases media-alta (blanca) de Lisboa también tiene prohibida su entrada en Pensão Amor. ${ }^{15}$

La "noche vintage" como estrategia de gentrificación del ocio nocturno en Cais do Sodré no pertenece tan sólo al ámbito de las reflexiones realizadas en este texto. El canal de la BBC-Travel informó recientemente que la Pensão Amor "once a brothel, [it] had been reborn as an art space with a bordello-chic bar" (Christiani, 2012). Etiquetar Pensão Amor como un burdel "chic" sin duda alguna refuerza la tesis expuesta hasta ahora en este texto acerca del papel gentrificador de dicho local. Por otra parte, el uso de este término sugeriría algunos cambios en el significado del término "bohemia". Por una parte, Pensão Amor ofrece un espacio de diseño de interior recordando los locales bohemios de las ciudades más activas en el ámbito de la cultura a lo largo de la década de los años 1920 y 1930, como por ejemplo Chicago (Cressey, 1932), Nueva York o el "París de América" (Burke, 1995), Barcelona (Villar, 1996) y Lisboa (Machado, 2008). Sin embargo, la re-significacion del término bohémia se referiría a la dimensión "conductual" del individuo el cual, si quiere participar del consumo de la noche elitista vintage, tiene que asumir los nuevos códigos de conducta asociados de la neobohemia: gestos y expresiones faciales mínimos, conversas despolitizadas, consumo de alcohol muy moderado y de marca, corrección absoluta en las formas de sentarse y/o de estar en la barra, del trato con el camarero y una preferencia por el pago con tarjeta bancaria, etc. ${ }^{16}$ Los individuos de esta bohoburguesía evitan expresiones en público reconocibles más allá de los límites del espacio íntimo creado en la mesa donde están sentados: el júbilo, la felicidad explícita, las carcajadas, el baile sensual ${ }^{17}$, el beber casi compulsivamente porque se tiene sed o porque sencillamente a uno le da la gana es inapropiado. Con todo, Pensão Amor ofrece un menú de elegancia en un contexto de ocio nocturno socialmente higienizado, tematizado, de placer predecible y fantasía controlada: ¿un ocio “Disneyzado", en términos de Alan Bryman (2004)?

\section{Conclusiones}

La popularidad de la Pensão Amor entre los jóvenes y jóvenes-adultos de las clases medias-altas de Lisboa demuestra el alto grado de resiliencia y fexiblidad del ocio nocturno glocal de pequeñas dimensiones y su importancia de primer orden en los procesos de gentrificación e higienización social de los centros históricos de la ciudad postindustrial. En este sentido, este artículo ha mostrado el caso de un ocio nocturno recientemente aparecido el cual, basado en la promoción de "lo vintage" como elemento de acumulación de capital cultural y distinción social, juega un papel fundamental en el proceso de gentrificación del antiguo barrio marinero de Cais do 
Sodré (Lisboa), sustituyendo progresivamente aquel ocio nocturno "auténtico" que caracterizaba las noches del "viejo" Cais por una nueva escena de la vida nocturna de marcada carácter bohemio-burgués. Sin lugar a dudas ello refuerza aún más la segregación socio-espacial del ocio nocturno de Lisboa cuyo estudio, hasta la fecha, carece de referentes bibliográficos.

21 Este artículo demuestra que las teorías sobre los diferenciales de renta y valor del suelo como paradigmas explicativos de la gentrificación son claramente insuficientes en algunos casos como, por ejemplo, en la exploración de cómo tal proceso de transformación urbana - en contextos de crisis financiera e inmobiliaria - penetran de forma selectiva en barrios populares degradados de los centro históricos mediante la renovación de un edificio abandonado y la generación de nuevas dinámicas cotidianas vinculadas a los estilos de vida y al consumo hedonista de ocio nocturno de las nuevas clases medias.

Además, si bien ambas teorías (rent-gap y value-gap, en original) pueden ser útiles en el análisis geográfico de la gentrificación en un período largo, son otra vez insuficientes en el análisis etnográfico del día a día del proceso gentrificador. Tal crítica epistemológica es la que subyace a lo largo del desarrollo de este artículo. De hecho, y debido en gran parte a la crisis financiera e inmobiliaria y la aparición de los primeros episodios de violencia urbana y malestar social durante estos últimos años, la gentrificación adopta nuevas formas, apareciendo de manera más fragmentada, en la que los agentes gentrificadores seleccionan aquellos edificios que constituirán las bases de lo que podría llamarse "gentrificación microcirúrgica". Se trata de una gentrificación de "baja intensidad" que está llamada a caracterizar los procesos de renovación urbana en esta nueva etapa de postcrisis. En ella, la "noche" global aparece como uno de los pilares fundamentales de la higienización social del espacio urbano así como del proceso de erradicación de elementos "peligrosos" para el proceso de internacionalización de la ciudad.

Este trabajo ha contado con el apoyo financiero de la Fundação para a Ciência e a Tecnologia de Portugal (FCT-SFRH/BPD/63178/2009) y el apoyo logístico del Centro de Estudos de Sociologia da Universidade Nova de Lisboa (CesNova). Quiero expresar mi más profundo agradecimiento al Profesor Marc Oliva, colega geógrafo del Instituto de Geografia e Ordenamento do Território da Universidade de Lisboa (IGOT-UL), por su ayuda, compañía, copas, ideas, reflexiones, críticas y puntos de vista innovadores en la realización de éste y tantos otros trabajos de campo de (en) la noche; también a Emilio Cambeiro, y a Adan Casán DJ El Matador, por sus respectivos puntos de vista "singulares" sobre la noche.

\section{BIBLIOGRAFIA}

BAPTISTA, L. (2005), “Territórios Lúdicos (e o que se torna lúdico um território): Ensaiando um ponto de partida”, Fórum Sociológico, 13-14 (2): 47-58.

BOURDIEU, P. (1979), La Distinction, Paris, Éditions de Minuit, Le Sens commun.

BRYMAN, A. (2004), The Disneyzation of Society, London, Sage. 
BURKE, P. (1995), “The invention of leisure in Early Modern Europe”, Past and Present, 146: 136-150.

BUTLER, T. (1997), Gentrification and the Middle Classes, Aldershot, Ashgate.

CHATTERTON, P., R. Hollands, B. Byrnes and C. Read (2002), The London of the North? Youth cultures, urban change and nightlife in Leeds, Case Study Report. Centre for Urban and Regional Development Studies, University of Newcastle.

CHATTERTON, P. and R. Hollands (2003), Urban Nightscapes. Youth Cultures, pleasure spaces and corporate power, London-New York, Routledge.

CHRISTIANI, K. (2012), “The rebirth of Lisbon's Cais do Sodré”, BBC-Travel Channel [Available on line at: http://www.bbc.com/travel/feature/20120320-the-rebirth-of-lisbons-cais-do-sodre, 24 March 2012, 1:47 pm].

CRESSEY, P. G. (1932), The Taxi-Dance Hall: A Sociological Study in Commercialized Recreation and City Life, Chicago, University of Chicago Sociological Series.

DAVIS, M. (1990), City of Quartz: Excavating the Future in Los Angeles, New York, Vintage Books. DURÃO, V. C. M. (2012), “Reclaimed Land: an Urban Analysis The landfills in Lisbon's downtown and riverfront", Journal of Integrated Coastal Zone Management (in press) [Avaliable online at: http://www.aprh.pt/rgci/pdf/rgci-288_Durao.pdf, 27 march 2012, 3:49 pm].

EDENSOR, T. (1998), Tourists at the Taj: Performance and Meaning at a Symbolic Site, New York, Routledge.

FEIXA, C. and J. Nofre (2012), "Youth Cultures". En Klandermans, B. (ed) Sociopedia, London, SAGE. GOTTDIENER, M. (2001), The Theming of America. American Dreams, Media Fantasies and Themed Environments, Boulder, CO, Westview Press.

HAEL, L. (2011), Dilemmas of the Nightlife Fix: Post-industrialization and the Gentrification of Nightlife in New York City, Urban Studies, 48(6):3449-3465.

HAMNETT, C. and B. Randolph (1984), The role of landlord disinvestment in housing market transformation: an analysis of the flat break-up market in central London. Transactions of the Institute of British Geographers, 9: 259-79.

HAMNETT, C. and B. Randolph (1986), "Tenurial transformation and the flat break-up market in London: the British condo experience". In N. Smith and P. Williams (eds) Gentrification of the City, Boston, Allen and Unwin, pp. 121-52.

HANNERZ, U. (1980), Exploring the City. Inquiries towards an Urban Anthropology, New York, Columbia University Press.

HANNIGAN, J. (1998), Fantasy City: Pleasure and Profit in the Postmodern Metropolis, New York, Routledge.

HOBBS, D., P. Hadfield, S. Lister and S. Winlow (2005), Bouncers: violence and governance in the nighttime economy, Oxford \& New York, Oxford University Press.

JUDD, D. R. (2003), El turismo urbano y la geografía de la ciudad, Revista Eure, 87 (29): 51-62.

LEES, L., T. Slater and E. Wyly (2008), Gentrification, New York, Routledge.

LEFEBVRE, H. (1968), Le droit à la ville, Paris, Ed. Anthropos.

LEY, D. (2003), "Artists, Aestheticisation and the Field of Gentrification", Urban Studies, 40(12):

2527-2544.

Forum Sociológico, 23 | 2013 
MENDES, L. (2006), “A nobilitação urbana no Bairro Alto: Análise de um processo de recomposição socioespacial”, Finisterra, XLI, 81: 57-82.

NOFRE, J. (2011), Youth Policies, Social Sanitation, and Contested Suburban Nightscape, in C. Perrone, G. Manella, L. Tripodi (ed.) Everyday Life in the Segmented City. Research in Urban Sociology, 11. Emerald Group Publishing, pp. 261-281.

NOFRE, J. (2010), “Políticas culturales, transformaciones urbanas e higienización social en la Barcelona contemporânea", Anales de Geografía de la Universidad Complutense de Madrid, 30(2): 133-61.

NOFRE, J. (2009a). L'Agenda Cultural Oculta. Una deconstrucció de l'oci nocturn de Barcelona i els seus suburbis. Premi Joventut 2009. PhD thesis. University of Barcelona [Available online at: http:// www.tdx.cat/handle/10803/1966, last access on: 3 May 2012, 5:26 pm].

NOFRE, J. (2009b), “Les politiques culturelles et de jeunesse à Barcelone et ses banlieues, un essai critique. Du la colonisation culturelle à l'homogénéisation social”, Sud-Ouest Européen: revue géographique des Pyrénées et du Sud-Ouest, 26(1): 83-96.

NOFRE, J. y J. Martín (2009), “Ocio nocturno, gentrificación y distinción social en el centro histórico de Sarajevo", Anales de Geografía de la Universidad Complutense de Madrid, 29(1): 91-110.

PAIS, J. M. (2008), A prostituição e a Lisboa Boémia. Lisboa: Âmbar.

PETÓNNET, C. (1982), “L'observation flottante. L'exemple d'un cimitière parisien”, L'Homme, 224: 23-35.

RITZER, G. (2010), Enchanting a Disenchanted World: Continuity and Change in the Cathedrals of Consumption, New York, Sage.

RUSSELL, H. (2008), Research Methods in Anthropology: Qualitative and Quantitative approaches, Oxford, Altamira Press.

SAVAGE, M. y T. Butler (1995), Social Change and the Middle Classes, London, Routledge.

SENNETT, R. (1970), The Uses of Disorder: Personal Identity and City Life, W.W. Norton, New York.

SILVA, C. (2011), "Cais do Sodré. Os novos inquilinos chegam à Pensão Amor e ao Bar da Velha Senhora", ionline, 10 November [Available online at: http://www.ionline.pt/boa-vida/cais-sodreos-novos-inquilinos-chegam-pensao-amor-ao-bar-da-velha-senhora, 22 march 2012, 5:14 pm].

SMITH, N. (1979), “Gentrification and Capital: Practice and Ideology in Society Hill”, Antipode, A Radical Journal of Geography, 3.2: 24-35.

TALBOT, D. (2004), "Regulation and Racial Differentiation in the Construction of Night-time Economies: A London Case Study”, Urban Studies, 41 (4):887-901.

TALBOT, D. (2006), "The Licensing Act 2003 and the Problematization of the Night-time Economy: Planning, Licensing and Subcultural Closure in the UK", International Journal of Urban and Regional Research, 30 (1):159-71.

TALBOT, D. (2007), Regulating the night: race, culture and exclusion in the making of the nighttime economy, Re-materialising cultural geography. Aldershot, England: Ashgate Pub. Company.

THOMAS, C. y R. Bromley (2000), “City-centre Revitalisation: Problems of Fragmentation and Fear in the Evening and Night-time City", Urban Studies 37(8), 1403-1429.

THORNTON, S. (1995), Club Cultures. Music, Media and Subcultural Capital, Cambridge, Polity Press. 
TRINDADE, S. (2011), “Hoje, beijo-te Lisboa”, Vogue, 17 Novembro 2011 [Available online at: http://www.vogue.xl.pt/lifestyle/sa\%C3\%83\%C2\%ADda-de-emerg\%C3\%83\%C2\%AAncia/2073hoje-beijo-te,-lisboa.html, 28 March 2012, 5:19 pm].

VILLAR, P. (1996), Historia y Leyenda del Barrio Chino (1900-1992). Cronica y Documentos de los Bajos Fondos de Barcelona, Barcelona, Ed. La Campana.

WYNNE, D. (1998), Leisure, Lifestyle and the New Middle Class. A case study, London, Routledge. WYNNE, D. y J. O'Connor (1998), “Consumption and the postmodern city”, Urban Studies, 35 (5-6): 841-64.

\section{NOTAS}

1. Como contribución destacada, Teresa Alves, de la Universidad de Lisboa, realizó en 2009 presentando una unidad didáctica acerca de la economía del ocio nocturno en Lisboa.

2. Para una detallada recopilación bibliográfica sobre los procesos de gentrificación en ciudades occidentales, véase Lees et al. (2008).

3. Rua do Norte, 81. Cerró sus puertas en otoño de 2010.

4. Fuente: http://spoiledmilk.lecool.com/lisboa/pt/2627?show_share_panel=true

5. Fuente: http://www.facebook.com/pages/Cais-Sodr\%C3\%A9-Cabaret/321365328077?sk=info [accessed on: 20 March 2012, 3:47 pm].

6. Información facilitada por J., 29 años, becario predoctoral de sociología urbana, residente de Cais de Sodré desde hace 7 años. Entrevista llevada a cabo in situ el 28 de Enero de 2012, alrededor de las 2:00 am en Pensão Amor. Entrevista registrada manualmente.

7. Kerry Christiani (2012).“The rebirth of Lisbon's Cais do Sodré”, BBC-Travel Channel. [http:// www.bbc.com/travel/feature/20120320-the-rebirth-of-lisbons-cais-do-sodre, 24 Marzo de 2012, $1: 47 \mathrm{pm}]$.

8. Fuente: "Cais do Sodré é sexy! Pensão Amor, Bar da Velha Senhora e O Povo: A troika de bares que veio arrasar a noite Lisboeta", Ruadebaixo, no. 76, Dezembro 2011 [http:// www.ruadebaixo.com/cais-do-sodre-sexy-como-nunca.html, 26 Marzo de 2012, 5:42 pm].

9. En Musicbox se realizan la mayor parte de Festivales de Música Underground que se celebran en Lisboa a lo largo del año.

10. Para más información, léase: http://bilioso.blogspot.pt/2011/12/recrudescem-as-mafiascorrupcao.html [08/07/2012, 12:24pm].

11. Información facilitada por L., 37 años, miembro de equipo de seguridad privada. Entrevista realizada in situ el 15 de Febrero de 2012, sobre las 0:30 am. La información no fue registrada por motivos de seguridad.

12. Idem.

13. Cfr. Nota 12 .

14. Cfr. Nota 8.

15. Conclusiones derivadas de la observación flotante realizada en el acceso inferior del local.

16. Conclusiones obtenidas a partir de la observación continuada a lo largo de varios días del momento de pagar de aquellos clientes que tuve la oportunidad de seguir visualmente el proceso. 17. "Muchos africanos y brasileños a veces bailan inadecuadamente, con bailes muy sexuales, con mucho contacto. A los que venimos aquí no nos gusta..." [traducido por el autor, original en portugués]. Declaraciones de J., 34 años, residente en Lisboa, en Alfama, software developer. Sus declaraciones son compartidas por C., 38 años, trabaja en una promotora inmobiliaria local; M., 30 años, becario posdoctoral, extranjero proveniente de un país de la UE. Entrevista realizada in situ, 12 de Noviembre de 2011, sobre las 2:15 am. Conversa registrada manualmente. 


\section{RESUMOS}

Ao longo das últimas três décadas, as estratégias culturais para a renovação urbana têm desempenhado um papel fundamental na transformação da cidade central. Junto com o consumo hedonista juvenil, a elitização do lazer noturno aparece como processo nuclear de renovação urbana (e social) em áreas urbanas degradadas dos centros históricos. Este artigo apresenta os primeiros resultados do trabalho de campo qualitativo sobre gentrificação e lazer noturno no velho bairro marinheiro do Cais do Sodré (Lisboa). Mais especificamente, este artigo tem como objetivo analisar como a conversão de uma pensão abandonada e frequentada por prostitutas "marginais" e marinheiros bêbados tem sido remodelada num local noturno elitista onde o consumo de "vintage" aparece como um mecanismo de acumulação de capital cultural e de distinção social, contribuindo significativamente no processo de gentrificação do lazer noturno do Cais do Sodré.

Over these last three decades, culture-led strategies of urban renovation have played a key role in several large-scaled urban transformations throughout Europe. Together with the emergence of youthful hedonist consumption, nightlife has become one of the most important strategies of urban renewal. Focusing on the Cais do Sodré neighborhood in Lisbon downtown, this paper aims to show how an abandoned hostel formerly frequented by "marginal" prostitutes has been transformed into a "vintage" club oriented to native-born new middle classes by favoring the rise of a "gentrified nightlife" in this central area of the Portuguese capital.

\section{ÍNDICE}

Palavras-chave: vintage, consumo hedonista juvenil, lazer noturno, gentrificação, Lisboa Keywords: vintage style, nightlife, gentrification, Lisbon

\section{AUTOR}

\section{JORDI NOFRE}

Centro de Estudos Sociológicos - UNL (CesNova). Faculdade de Ciências Sociais e Humanas Universidade Nova de Lisboa (jnofre@fcsh.unl.pt) 